\title{
Smoking signature is superior to programmed death-ligand 1 expression in predicting pathological response to neoadjuvant immunotherapy in lung cancer patients
}

\author{
Haitang Yang ${ }^{1 \#} \wedge$, Wenyan Ma ${ }^{2 \#}$, Beibei Sun ${ }^{3 \#}$, Liwen $\mathrm{Fan}^{1}, \mathrm{Ke} \mathrm{Xu^{1 }}$, Sean R. R. Hall ${ }^{4}$, \\ Mohammad Faisal Al-Hurani ${ }^{5}$, Ralph A. Schmid ${ }^{6}$, Ren-Wang Peng ${ }^{6}$, Toyoaki Hida ${ }^{7}$, Zhexin Wang $^{1}$, Feng Yao ${ }^{1}$ \\ ${ }^{1}$ Department of Thoracic Surgery, Shanghai Chest Hospital, Shanghai Jiao Tong University, Shanghai, China; ${ }^{2}$ Clinical Research Center, Shanghai \\ Chest Hospital, Shanghai Jiao Tong University, Shanghai, China; ${ }^{3}$ Institute for Thoracic Oncology, Shanghai Chest Hospital, Shanghai Jiao Tong \\ University, Shanghai, China; ${ }^{4}$ Wyss Institute for Biologically Inspired Engineering, Harvard University, Boston, MA, USA; ${ }^{5}$ Department of General \\ and Special Surgery, Faculty of Medicine, The Hashemite University, Zarqa, Jordan; ${ }^{6}$ Division of General Thoracic Surgery, Department of \\ BioMedical Research (DBMR), Inselspital, Bern University Hospital, University of Bern, Bern, Switzerland; ${ }^{7}$ Lung Cancer Center, Central Japan \\ International Medical Center, Minokamo, Gifu, Japan \\ Contributions: (I) Conception and design: H Yang, W Ma, F Yao; (II) Administrative support: Z Wang, F Yao; (III) Provision of study materials or \\ patients: B Sun, L Fan, K Xu; (IV) Collection and assembly of data: B Sun, L Fan, K Xu; (V) Data analysis and interpretation: W Ma, B Sun, L Fan, \\ $\mathrm{K} \mathrm{Xu}$; (VI) Manuscript writing: All authors; (VII) Final approval of manuscript: All authors. \\ \#These authors contributed equally to this work. \\ Correspondence to: Feng Yao; Zhexin Wang. Department of Thoracic Surgery, Shanghai Chest Hospital, Shanghai Jiao Tong University, West Huaihai \\ 241, Shanghai 200030, China. Email: feng.yao@shchest.org; wzx1953@shchest.org.
}

\begin{abstract}
Background: There is a paucity of biomarkers that can predict the degree of pathological response [e.g., pathological complete response (pCR) or major response (pMR)] to immunotherapy. Neoadjuvant immunotherapy provides an ideal setting for exploring responsive biomarkers because the pathological responses can be directly and accurately evaluated.

Methods: We retrospectively collected the clinicopathological characteristics and treatment outcomes of non-small cell lung cancer (NSCLC) patients who received neoadjuvant immunotherapy or chemoimmunotherapy followed by surgery between 2018 and 2020 at a large academic thoracic cancer center. Clinicopathological factors associated with pathological response were analyzed.

Results: A total of 39 patients (35 males and 4 females) were included. The most common histological subtype was lung squamous cell carcinoma (LUSC) ( $\mathrm{n}=28,71.8 \%$ ), followed by lung adenocarcinoma (LUAD) ( $\mathrm{n}=11$, 28.2\%). After neoadjuvant treatment, computed tomography (CT) scan-based evaluation showed poor agreement with the postoperatively pathological examination (weighted kappa $=0.0225 ; \mathrm{P}=0.795$ ), suggesting the poor performance of CT scans in evaluating the response to immunotherapy. Importantly, we found that the smoking signature displayed a better performance than programmed death-ligand 1 (PD-L1) expression in predicting the pathological response (area under the curve: 0.690 s. 0.456; $\mathrm{P}=0.0259$ ), which might have resulted from increased tumor mutational burden (TMB) and/or microsatellite instability (MSI) relating to smoking exposure.

Conclusions: These findings suggest that CT scan-based evaluation is not able to accurately reflect the pathological response to immunotherapy and that smoking signature is a superior marker to PD-L1 expression in predicting the benefit of immunotherapy in NSCLC patients.
\end{abstract}

Keywords: Neoadjuvant immunotherapy; lung cancer; smoking; pathological response; biomarker

Submitted May 28, 2021. Accepted for publication Sep 16, 2021.

doi: $10.21037 /$ tlcr-21-734

View this article at: https://dx.doi.org/10.21037/tlcr-21-734

^ORCID: 0000-0002-8732-5910. 


\section{Introduction}

Immune checkpoint inhibitors (ICIs), either alone or in combination with platinum doublet-based chemotherapy, have shown survival benefits for patients with metastatic non-small cell lung cancer (NSCLC) (1,2). Currently, three biomarkers, namely programmed death-ligand 1 (PD$\mathrm{L} 1)$, tumor mutational burden (TMB), and microsatellite instability (MSI), have been clinically approved to guide the management of ICIs for patients with various cancer types. In particular, PD-L1 is routinely and widely used due to its clinical practicability. Despite these biomarkers being available for patient stratification, clinical responses vary substantially among patients, suggesting that additional biomarkers are needed to identify patient subsets that can truly and precisely benefit from ICI treatment. Concerning another two clinically-approved biomarkers TMB and MSI, the determination of TMB/MSI was complicated and did not reach a good consensus in terms of the clinical application (3). Moreover, ICIs incur high costs and show selective and heterogeneous efficacy (4). Therefore, the identification of biomarkers that can predict response to ICIs and enable the selection of the most suitable subpopulations is of critical importance.

Another critical issue is that previous studies have mainly relied on the radiographic evaluation [e.g., computed tomography $(\mathrm{CT})]$ of the therapy response to ICIs, although this cannot truly reflect the pathological response, as evidenced by research in neoadjuvant chemotherapy (5). This leads to the inability to identify those subsets that would most benefit from ICIs, subsequently affecting treatment decision making. Given this, patients may not receive optimal treatment regimens. Given the poor performance of traditional radiographic-based evaluation, a more complex system has been recently proposed, such as quantitative immune-related pathological response criteria [e.g., (I) immune activation-dense tumour-infiltrating lymphocytes with macrophages and tertiary lymphoid structures (TLSs); (II) massive tumor cell death-cholesterol clefts; and (III) tissue repair-neovascularization and proliferative fibrosis] (6) or a rigorous protocol for tissue sample harvesting and processing [e.g., (I) viable tumor, (II) necrosis, and (III) stroma (including inflammation and fibrosis)] (7). Notably, neoadjuvant immunotherapy provides an ideal setting for exploring predictive biomarkers because the pathological responses can be directly and accurately evaluated. Recent attention has been focused on exploring ICIs as a neoadjuvant treatment strategy, followed by surgery, in locally advanced NSCLC, and this strategy has demonstrated early clinical promise (8-12). Despite these advances, there is still a lack of actionable biomarkers that can predict the pathological response to ICIs (13).

In this retrospective study, we analyzed the clinicopathological data of locally advanced NSCLC patients who had received surgery after neoadjuvant immunotherapy or chemo-immunotherapy. Our data showed that CT had a poor performance in evaluating the treatment response in neoadjuvant settings. Of note, we found that smoking history but not PD-L1 expression is associated with a high pathological response to ICIs, which might be due to a smoking-related increase in TMB and/ or MSI. Thus, we identified the smoking signature as an additional reasonable and actionable biomarker for the management of immunotherapy for lung cancer patients.

We present the following article in accordance with the STARD reporting checklist (available at https://dx.doi. org/10.21037/tlcr-21-734).

\section{Methods}

\section{Study design and patients}

Clinical records of NSCLC patients undergoing surgery between 2018 and 2020 at Shanghai Chest Hospital were retrospectively reviewed. Clinical data (demographic data, clinical and pathological characteristics, therapeutic regimens, and clinical outcomes) of lung cancer patients who received neoadjuvant immunotherapy or chemoimmunotherapy were retrieved for further analysis. This study was approved by the institutional review board of Shanghai Chest Hospital [\#KS $(Y) 21039]$. All patients had signed informed consent for inclusion of their clinical data and specimens in our Lung Biobank and use in research projects, according to the recommendation of the ethical committee of Shanghai Chest Hospital. The study was conducted in accordance with the Declaration of Helsinki (as revised in 2013).

\section{Preoperative examinations}

Patients received preoperative assessment including brain magnetic resonance imaging (MRI), contrast-enhanced chest CT scan or positron emission tomography (PET)/ CT scan, bronchoscopy, abdominal CT or ultrasonography examination, and/or whole-body bone scan. The pathological confirmation based on tumor biopsies, PET- 
CT, or invasive mediastinoscopy [or endobronchial ultrasound-guided transbronchial needle aspiration (EBUSTBNA)] was used to evaluate mediastinal nodal staging. All patients had an Eastern Cooperative Oncology Group performance status (ECOG PS) of 0 to 1 , normal organ function, and sufficient lung function for resection.

NSCLC with targetable driver mutations, such as epidermal growth factor receptor $(E G F R)$, echinoderm microtubule-associated protein-like 4-anaplastic lymphoma kinase fusion oncogene (EML4-ALK), repressor of silencing 1 (ROS1), or mesenchymal-epithelial transition factor (MET), were routinely excluded for immunotherapy. Cancer serum biomarkers, including carcinoembryonic antigen (CEA), squamous cell carcinoma (SCC) antigen, fragment of cytokeratin subunit 19 (CYFRA 21-1), and neuron-specific enolase (NSE), were clinically and routinely used in our medical center for the diagnosis of lung cancer. Given that neutrophil to lymphocyte ratio (NLR) has been associated with the response to immunotherapy and survival (14), we also included NLR in our analysis.

\section{Neoadjuvant immunotherapy}

Neoadjuvant therapy-related information, such as agents, courses, doses, and duration of final neoadjuvant treatment to surgery, was collected. For patients receiving ICI alone, one of the following approved ICIs was administered: (I) pembrolizumab [Keytruda (Merck), antiprogrammed cell death protein 1 (PD-1), at a dose of 200 $\mathrm{mg}$ intravenously every 3 weeks]; (II) nivolumab [Opdivo (Bristol-Myers Squibb), anti-PD-1, at a dose of $240 \mathrm{mg}$ intravenously every 2 weeks]; (III) sintilimab [anti-PD-1; (jointly developed by Innovent Biologics and Eli Lilly), at a dose of $200 \mathrm{mg}$ intravenously every 3 weeks]; (IV) or ipilimumab [Yervoy (Bristol-Myers Squibb), anti-cytotoxic T-lymphocyte-associated protein 4 (anti-CTLA-4), at a dose of $3 \mathrm{mg}$ per kilogram, intravenously every 3 weeks]. For patients receiving chemo-immunotherapy, a single ICI plus platinum doublets were given. Patients received the first dose of immunotherapy concurrently with chemotherapy, and the subsequent doses were administered every 2 or 3 weeks (according to the corresponding indications) in a total of 2 to 5 cycles, and chemotherapy was given every 3 weeks in a total of 2 to 3 cycles. For patients receiving ICIs alone, PD-L1 expression with tumor proportion score (TPS) $\geq 50 \%$ was routinely applied, while chemo-immunotherapy was given irrespective of the PD-L1 TPS expression.

\section{Operation}

Posterolateral thoracotomy (open) or video-assisted thoracic surgery (VATS) with systemic lymphadenectomy was performed based on the patient's conditions, patients' informed consent, and surgeons' preference. The operative approach, the extent of resection, operative time, hospital length of stay, postoperative morbidity, and other details related to the operative experience, were recorded in detail. Clinical and pathological staging of patients were evaluated according to The American Joint Committee on Cancer (AJCC) Lung Cancer Staging (8th edition) (15). Surgical complications were documented according to the criteria defined by the Society of Thoracic Surgeons and the European Society of Thoracic Surgeons general thoracic surgery databases (16).

All patients received postoperative adjuvant immunotherapy or chemotherapy (with or without radiation) after discussion with the multidisciplinary tumor board was carried out.

\section{Treatment response assessments}

After completion of neoadjuvant treatment, PET/CT or CT scan was performed to evaluate therapeutic response and resectability, preferably 2 weeks after the last dose of therapy. The response was assessed based on the Response Evaluation Criteria in Solid Tumors (RECIST; version 1.1) (17). Later, patients who did not have disease progress underwent surgery in 5 to 6 weeks. Pathological complete response (pCR) was defined as the absence of viable tumor cells (ypT0N0M0) in the surgical resection specimen; pathological major response (pMR) was defined as $10 \%$ or less viable tumor cells in the surgical resection specimen; pathological partial response (pPR) was defined as 10$50 \%$ viable tumor cells in the surgical resection specimen; pathological small response (pSR) was defined as more than $50 \%$ viable tumor cells in the surgical resection specimen. The pathological response was graded according to the Junker criteria and assessed in all resected specimens (18).

\section{Smoking and PD-L1 subgroups}

Cumulative smoking exposure was determined in terms of pack-years by multiplying the number of years smoked with the average number of packs per day (19). Based on pack-years of smoking, participants were classified as never smokers (0.0 pack-years), light smokers (0.1-20.0 packyears), moderate smokers (20.1-40.0 pack-years), and heavy 
smokers (>40 pack-years) (19).

Tumoral PD-L1 expression was scored by a senior pathologist according to the PD-L1 tumor proportions score before neoadjuvant immunotherapy, and samples were divided into 3 subgroups: high ( $\geq 50 \%)$, moderate $(1-50 \%)$, and low $(<1 \%)$ PD-L1.

\section{Immunobistochemistry}

Serial $5-\mu \mathrm{m}$ tissue sections from biopsies and the matched resected primary tumors were deparaffinized, rehydrated, pretreated for antigen retrieval, and then stained with hematoxylin and eosin and subjected to immunohistochemical staining following a standard protocol (20). The following primary antibodies were used: anti-human PD-L1 (DaLo, monoclonal mouse antihuman, clone 22C3), cytokeratin 5/6 (CK5/6), P40, thyroid transcription factor 1 (TTF-1), Napsin A, and CD56.

\section{Public databases}

Clinical and genomic data of The Cancer Genome Atlas (TCGA) NSCLC cohort were extracted from the publicly available cBioPortal (https://www.cbioportal.org/) and were then integrated to analyze TMB (defined as the number of somatic, coding, base substitution, and indel mutations per megabase of genome examined) and MSI [based on the MANTIS method (21)]. For NSCLC patients receiving ICIs, the Memorial Sloan Kettering Cancer Center (MSKCC) (TMB and immunotherapy) cohort was mined (22).

\section{Follow-up}

The first follow-up visit was scheduled 4 weeks after discharge. Adjuvant therapies were then typically started 1 month postoperatively. Later, follow-up visits were scheduled every 3 months with chest CT scans, brain MRI, abdominal sonography or CT, and serum tumor markers (23). Other examinations were performed according to the oncologists when necessary. Followup information was obtained from patients by telephone calls or clinic revisit records.

Recurrence-free survival (RFS) was defined as the interval between the day of pathological diagnosis and the date of detected tumor relapse by any cause or the last follow-up date. Overall survival (OS) was defined as the interval between the day of pathological diagnosis and the date of death by any cause or the last follow-up date (March 2021).

\section{Statistical analysis}

Normally distributed continuous variables are presented as mean $\pm \mathrm{SD}$; otherwise, they are presented as median and range (23). Categorical variables are shown as numbers and percentages. Baseline characteristics were compared between tumors with $\mathrm{pCR} / \mathrm{p} M R$ or non-pCR/pMR cohorts by using chi-square or Fisher's exact test (expected frequencies <5) for categorical data. Multivariate logistic regression [by generalized linear model (GLM)] function in R (The R Foundation for Statistical Computing)] was used to identify predictors for pCR. Survival analysis was performed using "survminer" and "survival" R packages. Tumor samples were divided into 2 groups based on the best-separation cutoff value of TMB to plot the Kaplan-Meier survival curves. Kappa test was performed for agreement between CTbased evaluation and final histopathological examination of treatment response using the function "kappa2" from the package "irr" in R. Data summary and statistical analysis were performed using $\mathrm{R}$ software (version 3.6.3). A $\mathrm{P}$ value $<0.05$ was considered statistically significant.

\section{Results}

\section{Clinical characteristics}

A total of 39 patients who received neoadjuvant immunotherapy or chemo-immunotherapy were included in this study. The clinical characteristics of patients and tumors included in this study cohort are shown in Table 1. The patients' mean age at the time of the diagnosis was $60.49 \pm 7.58$ years, with 35 of the patients being males $(89.7 \%)$ and the other 4 being females $(10.3 \%)$. The majority (28 of 35 ) of males but not females had a smoking history, with $20.5 \%$ of males being heavy smokers.

The most common histological subtype was lung squamous cell carcinoma (LUSC) $(n=28,71.8 \%)$, followed by lung adenocarcinoma (LUAD) ( $\mathrm{n}=11,28.2 \%)$. Overall, the majority of the patients had a clinical stage (c-stage) IIIA disease $(22,56.4 \%)$, followed by stage IIIB ( $n=8,20.5 \%)$, stage IIB $(n=5,12.8 \%)$, stage IIA $(n=2,5.1 \%)$, and stage IB $(n=1,2.6 \%)$ disease at the initial diagnosis.

\section{Neoadjuvant immunotberapy or chemo-immunotberapy}

None of the patients received preoperative radiotherapy. 
Table 1 Clinicopathological characteristics

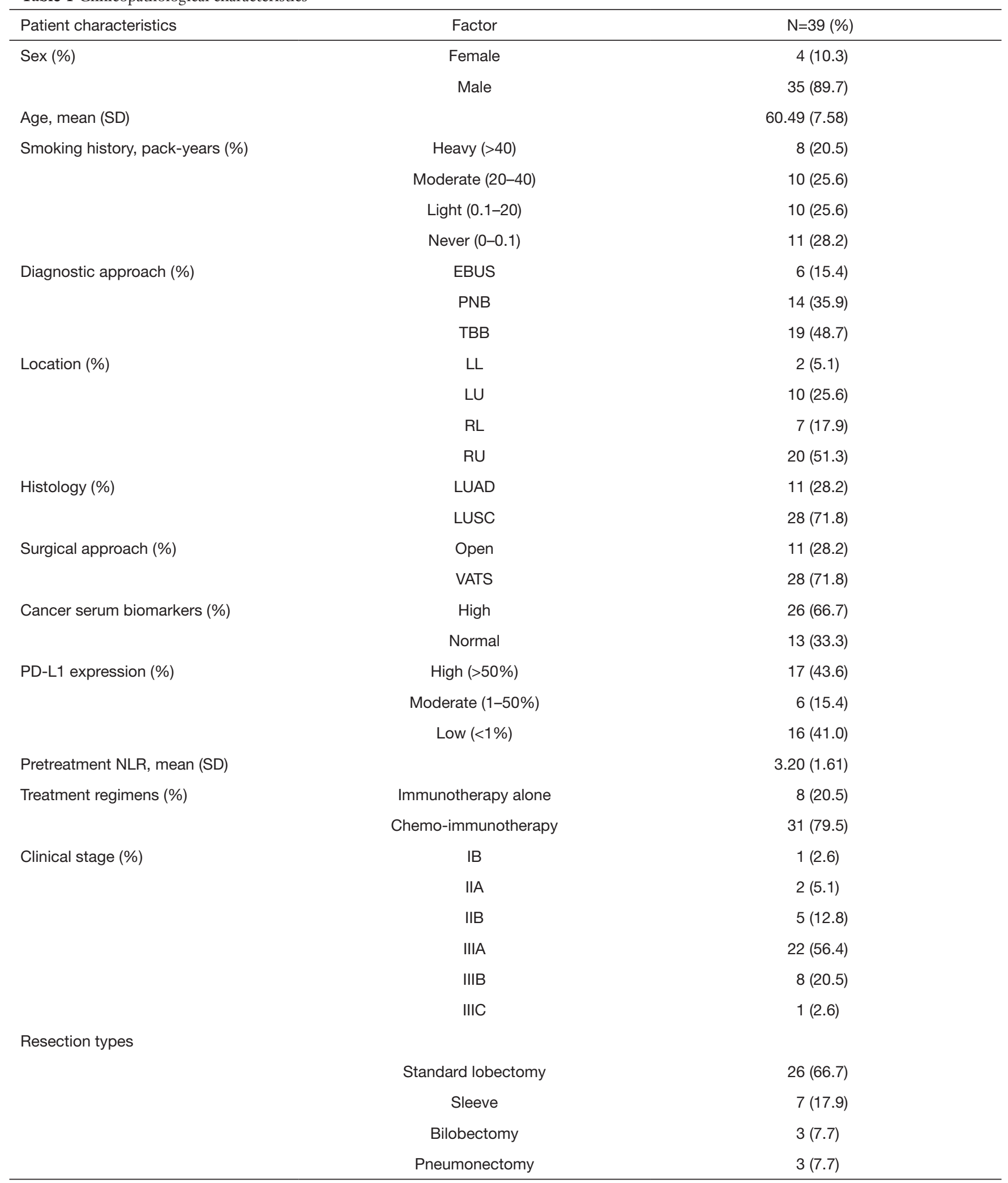

Table 1 (continued) 
Table 1 (continued)

\begin{tabular}{lcc}
\hline Patient characteristics & Factor & $\mathrm{N}=39(\%)$ \\
\hline Resection margin (\%) & Negative & $36(92.3)$ \\
& Positive & $3(7.7)$ \\
Pathological response (\%) & pCR & $13(33.3)$ \\
& pMR & $8(20.5)$ \\
& pPR & $12(30.8)$ \\
\hline
\end{tabular}

EBUS, endobronchial ultrasound; PNB, percutaneous needle biopsy; TBB, transbronchial biopsy; LL, left lower; LU, left upper; RL, right lower; RU, right upper; LUAD, lung adenocarcinoma; LUSC, lung squamous cell carcinoma; VATS, cideo-assisted thoracoscopic surgery; $\mathrm{pCR}$, pathological complete response; pMR, pathological major response; pPR, pathological partial response; pSR, pathological small response; NLR, neutrophil to lymphocyte ratio; PD-L1, programmed death-ligand 1.

The treatment regimens involving immunotherapy are summarized in Table S1. Patients received a median of 3 doses before resection (range, $2-5$ doses), 8 patients $(20.5 \%)$ received neoadjuvant immunotherapy alone, including single $(\mathrm{n}=7)$ and double $(\mathrm{n}=1)$ ICIs, and 31 patients $(79.5 \%)$ were managed with neoadjuvant chemo-immunotherapy.

Immunotherapy/chemo-immunotherapy related adverse events occurred in 15 patients with grade $3(\mathrm{n}=5)$ or higher $(\mathrm{n}=1)$ pneumonia, liver dysfunction $(\mathrm{n}=3)$, slight-tomoderate bone marrow suppression $(n=2)$, and others $(n=4)$. The median interval between final treatment to surgery was 36 days (range, 27-93 days), without treatment-related delay in surgery.

After neoadjuvant treatment, posttreatment CT scans showed that 29 patients $(74.4 \%)$ had a partial response and 10 patients $(25.6 \%)$ had stable disease.

\section{Surgical resection and postoperative course}

The most common resection type was standard lobectomy (26, 66.7\%), followed by sleeve lobectomy (7, 17.9\%), bilobectomy (3, 7.7\%), and pneumonectomy (3, 7.7\%). Minimally invasive approaches were performed in $28(71.8 \%)$ patients. Finally, radical surgery was achieved in 36 of $39(92.3 \%)$ patients. The positive surgical margin was mainly due to the limited lung function.

The median hospital postoperative stay was 7 days (range, 2-29 days). One or more postoperative manageable complications occurred in 18 out of 39 patients. The most common complication was prolonged air leak $(n=14)$, followed by arrhythmia $(n=4)$, pneumonia $(n=2)$, chylothorax $(\mathrm{n}=1)$, wound infection $(\mathrm{n}=1)$, and bronchopleural fistula $(n=1)$. There was one surgery-related patient death on day 29 postoperatively, which occurred due to bronchopleural fistula.

\section{Factors associated with pathological response}

Final pathological examination indicated that 8 of 39 patients (20.5\%) experienced MPR and that 13 patients $(33.3 \%)$ experienced pCR without residual viable tumor (Table 1).

In an attempt to identify the baseline characteristics that were associated with the high response (pCR plus pMR) to immunotherapy or chemo-immunotherapy, we found that, intriguingly, a heavy smoking history but not PDL1 expression or NLR was significantly related to a high pathological response (Table 2; Figure 1A-1E). Detailed analysis revealed that all the tumors from heavy smokers had a pCR or pMR in response to neoadjuvant treatment (Figure 1A-1C). Currently, PD-L1, TMB (24), and MSI (25) represent the only clinically approved biomarkers predicting the therapeutic benefits of ICIs. Smoking could induce an increased rate of mutated proteins or neoantigens in lung cancer (26-28). In support of this, mining of TCGA lung cancer cohort showed that heavy smoking is associated with high TMB (26-28) and MSI (29,30) (Figure 1F), which could partially explain the high degree of response in the heavy smokers. Along the same lines, univariate and multivariate analyses showed that high TMB was significantly associated with immunotherapy in NSCLC patients (Figure 1G). Additionally, we observed that there was no significant difference in the response rate between neoadjuvant immunotherapy and chemo-immunotherapy (Table 2). Furthermore, multivariate logistic regression analysis also identified heavy smokers as a significant predictor for $\mathrm{pCR}$ 
Table 2 Association between clinical characteristics and CR/MPR

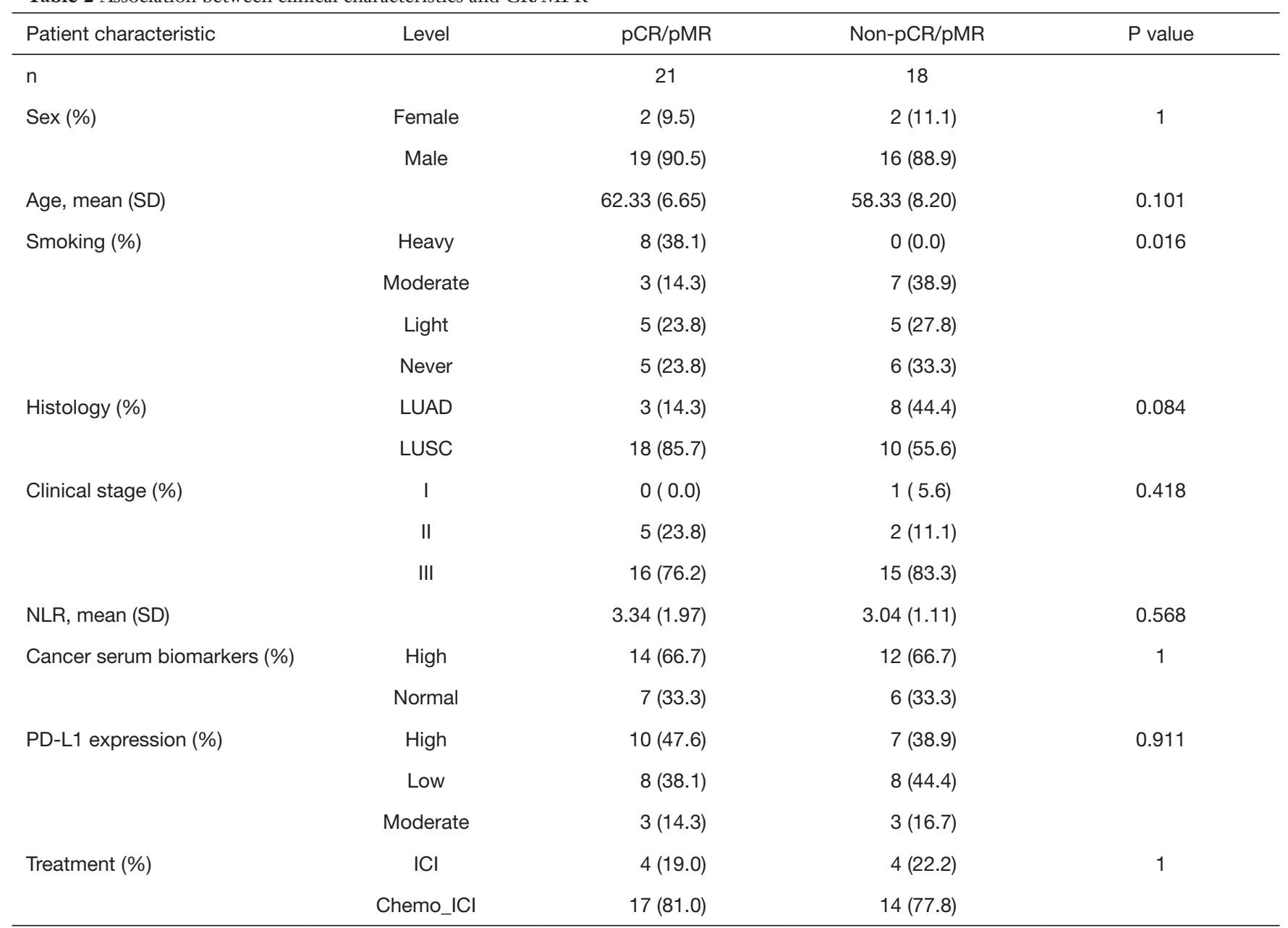

pCR, pathological complete response; pMR, pathological major response; LUAD, lung adenocarcinoma; LUSC, lung squamous cell carcinoma; NLR, neutrophil to lymphocyte ratio; PD-L1, programmed death-ligand 1; ICI, immune checkpoint inhibitor; Chemo_ICI, chemo-immunotherapy.

A

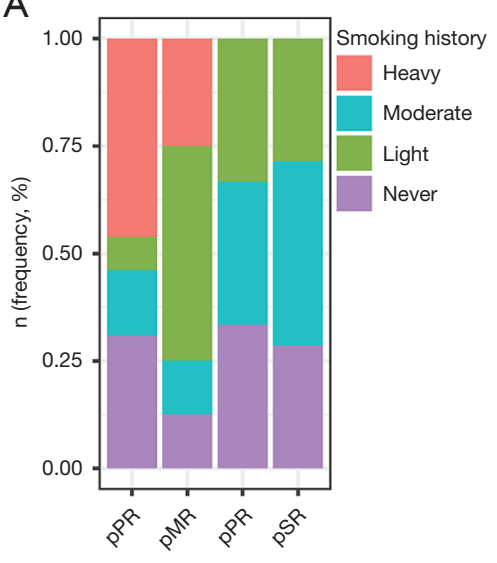

B

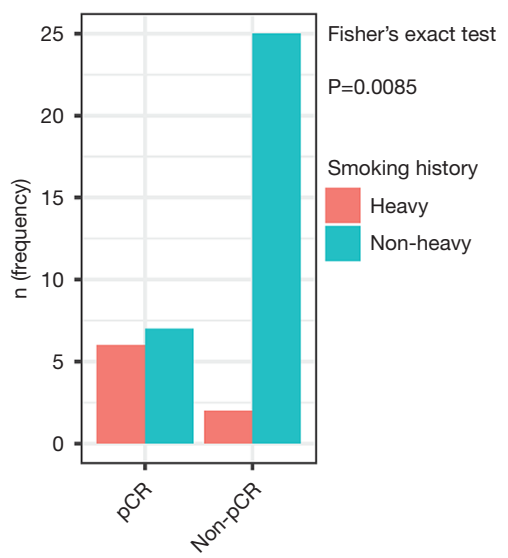

C

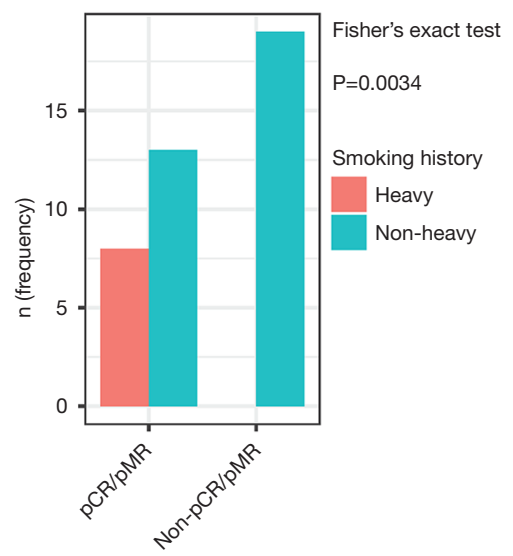


D



$E$

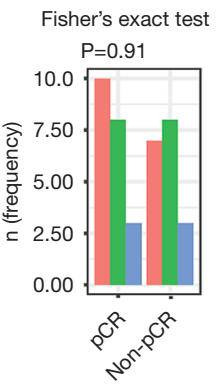

$\mathrm{F}$

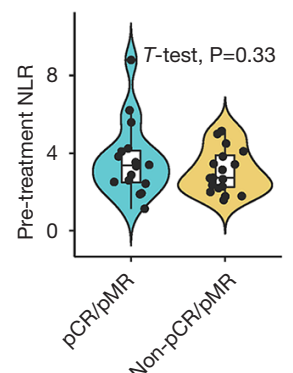

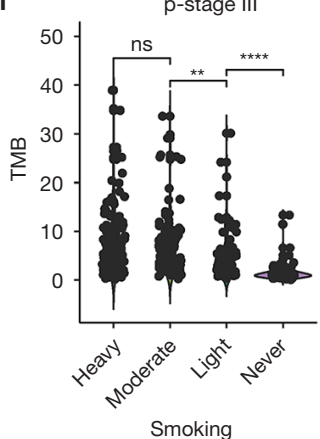

p-stage III

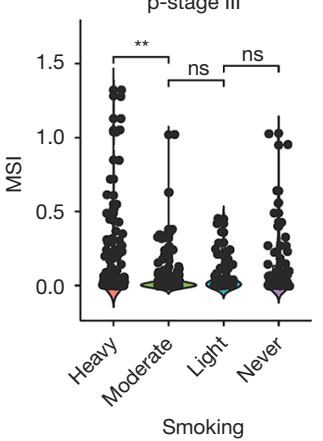

G

TMB and immunotherapy (MSKCC, nat genet 2019)



$\mathrm{H}$

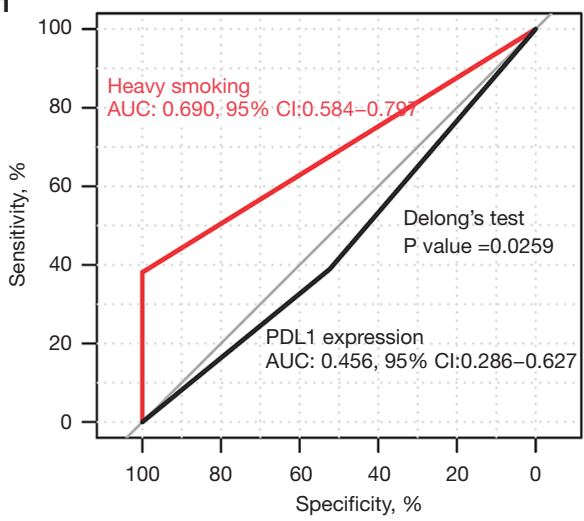



Figure 1 Smoking signature but not PD-L1 expression predicts pathological response to neoadjuvant immunotherapy. (A-C) Distribution of smoking history in NSCLC patients with different pathological responses to neoadjuvant immunotherapy. (D) The association between PD-L1 expression and pathological responses to neoadjuvant immunotherapy. (E) The association between NLR and pathological responses to neoadjuvant immunotherapy. (F) The association of smoking history with TMB and MSI in TCGA stage-III NSCLC. **, P<0.01; ***, $\mathrm{P}<0.0001$ by one-way Anova test. ns, not significant $(\mathrm{P}>0.05)$. (G) Univariate (left) and multivariate (right) analyses showing the association between TMB level and survival in patients receiving ICIs. Data were mined from the MSKCC TMB and immunotherapy cohort (Samstein RM, Nat Genet 2019). (H) ROC curve analysis the ability of heavy smoking exposure to identify responders (pMR/pCR). Sensitivity refers to the proportion of true positive subjects with the disease among all subjects with the disease. Specificity refers to the proportion of true negative subjects without the disease among subjects without the disease. PD-L1, programmed death-ligand 1; NSCLC, non-small cell lung cancer; NLR, neutrophil to lymphocyte ratio; TMB, tumor mutational burden; MSI, microsatellite instability; TCGA, The Cancer Genome Atlas; ICI, immune checkpoint inhibitor; MSKCC, Memorial Sloan Kettering Cancer Center; ROC, receiver operating characteristic; pCR, pathological complete response; pMR, pathological major response; pPR, pathologic partial response; pSR, pathological small response. 
Table 3 Multivariate logistic regression identifies predictors for pCR

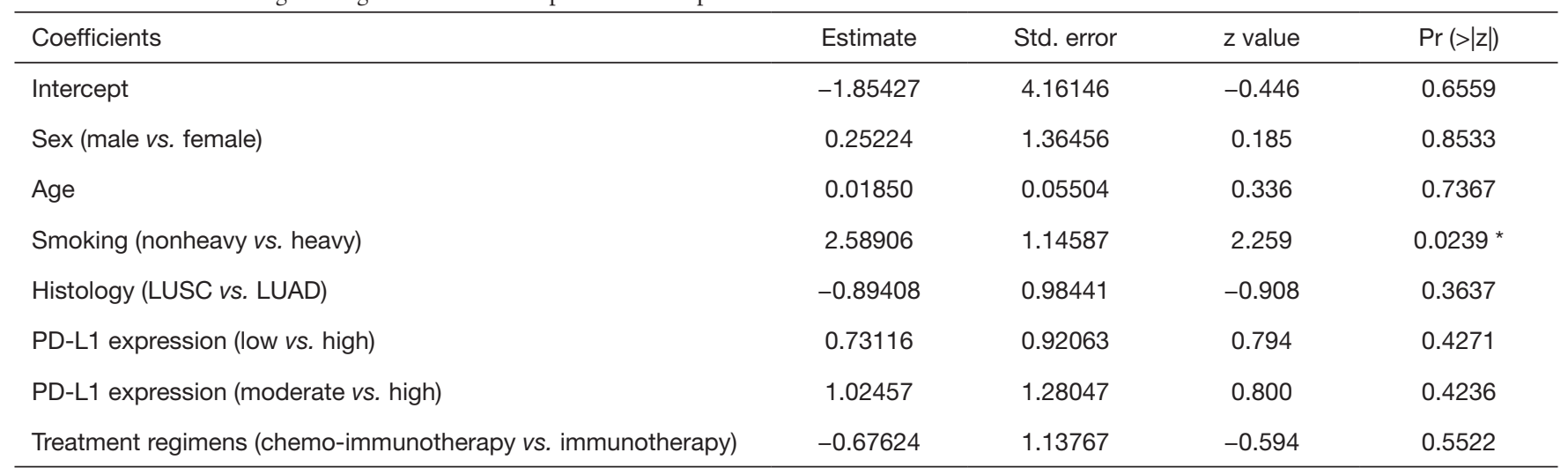

*, $\mathrm{P}<0.05$. pCR, pathological complete response; LUAD, lung adenocarcinoma; LUSC, lung squamous cell carcinoma; PD-L1, programmed death-ligand 1.

Table 4 Agreement analysis between CT and histopathological examination in evaluating pathological response to immunotherapy

\begin{tabular}{lccc}
\hline Response & CT scan & Pathology & Outcome \\
\hline SD & $10(25.6 \%)$ & 0 & Weighted kappa $=0.0225$ \\
PR & $29(74.4 \%)$ & $26(66.7 \%)$ & P value $=0.795$ \\
CR & 0 & $13(33.3 \%)$ & \\
\hline
\end{tabular}

CT, computed tomography; SD, stable disease; PR, partial response: CR, complete response.

(Table 3). Finally, receiver operating characteristic (ROC) analysis revealed that smoking signature was associated with a greater response to immunotherapy, compared with that of PD-L1 expression [area under the curve (AUC) 0.690, $95 \%$ confidence interval (CI): 0.584-0.797 vs. AUC 0.456, 95\% CI: 0.286-0.627; $\mathrm{P}=0.0259$; Figure $1 H]$.

\section{CT scan performance}

CT scan is routinely used to evaluate the treatment response. However, we observed a dramatic difference between CT scan assessment and the final histological examination in patients who received neoadjuvant immunotherapy (weighted Kappa $=0.0225 ; \mathrm{P}=0.795$; Table 4; Figure 2; Figure S1). In patients with pCR, 11 out of 13 still had a mass in the original tumor bed; however, postoperative pathological examination showed an absence of tumors being replaced with massive necrosis and infiltrative lymphocytes (Figure 2). These observations suggest that CT scan-based evaluation cannot accurately reflect the real treatment response and that biomarkers that can accurately predict the therapeutic response to ICIs are urgently needed.

\section{Survival}

The median follow-up time was 537 days (range, 217984 days). At the end of follow-up, 7 (59.2\%) patients had tumor relapse and 2 patients died. Two-year RFS and OS were $81.5 \%$ and $94.1 \%$, respectively (Figure 3).

\section{Discussion}

Despite the considerable success of immunotherapy in lung cancer, there is a lack of responsive biomarkers that can predict which patients will show pathological responses.

\section{The rationale of neoadjuvant immunotherapy for lung cancer}

The cytolytic activity of host cluster of differentiation (CD) 8 and CD4 T cells occurs following recognition of the tumor antigen presented on major histocompatibility complexes (MHC) I or MHC II, respectively. Thus, the neoantigen burden is regarded as a fundamental determinant of response to immunotherapy (31). In support of this, TMB has been approved as a PD-L1-independent biomarker 
A
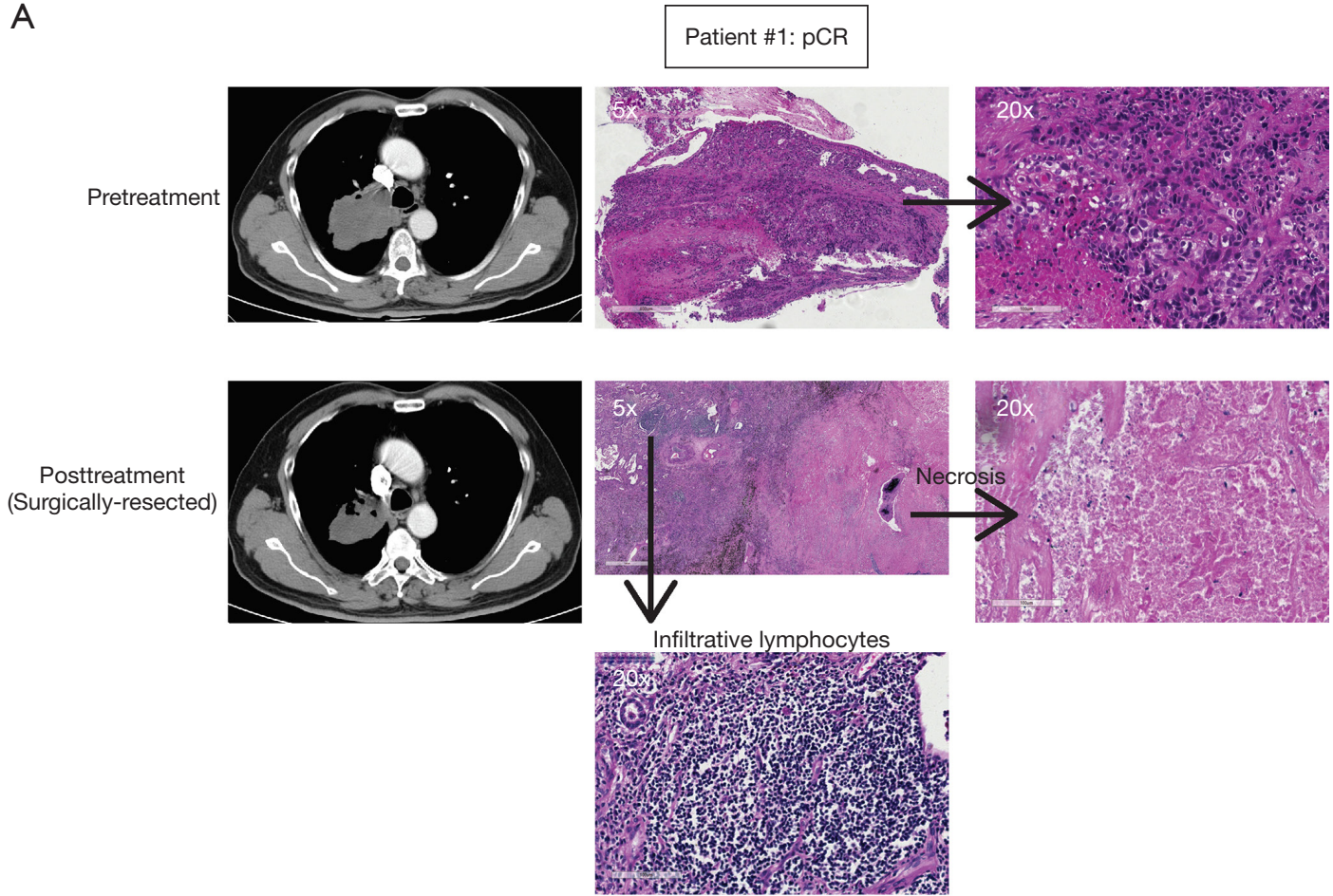

B

Patient \#2: pCR
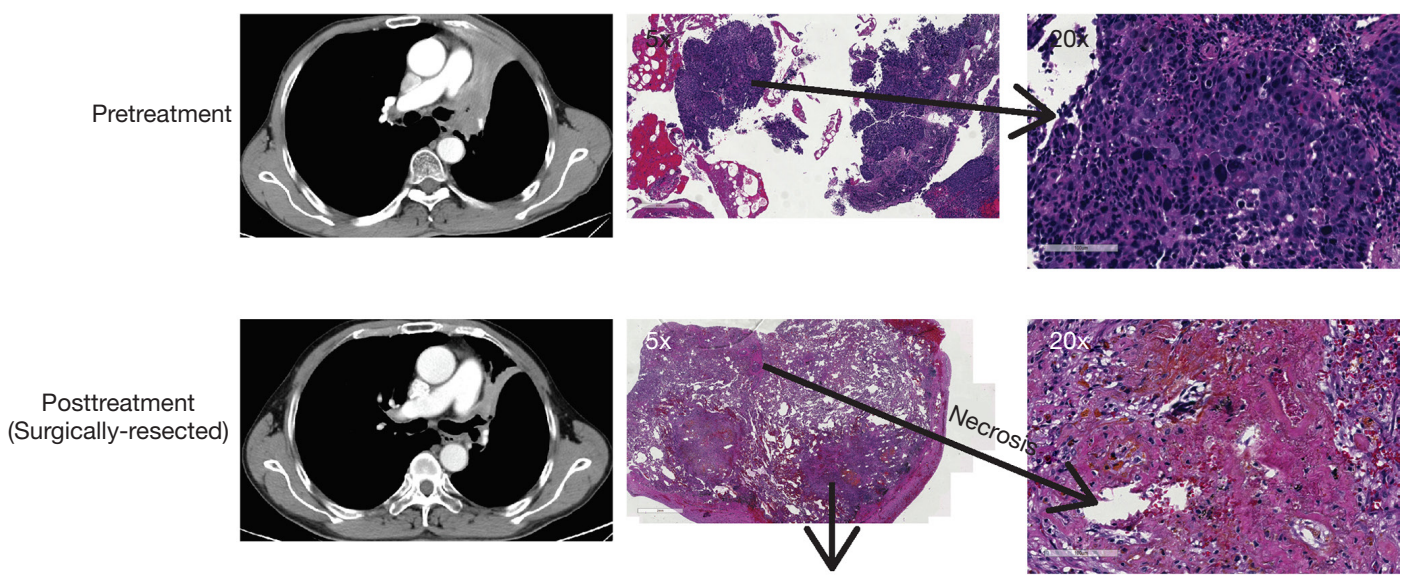

Infiltrative lymphocytes

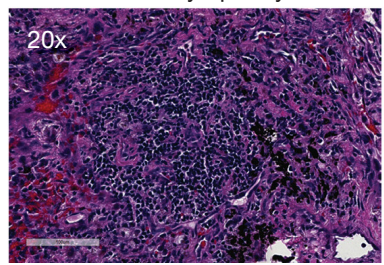

Figure 2 Poor performance of CT scans in evaluating pathological response to immunotherapy. (A,B) Two representative cases with pCR. Preoperative contrast-enhanced CT scan (within 1 week before surgery) and hematoxylin and eosin staining (magnifications: $50 \times$ and $200 \times$ ) of the resected samples are shown. CT, computed tomography; pCR, pathological complete response. 
A

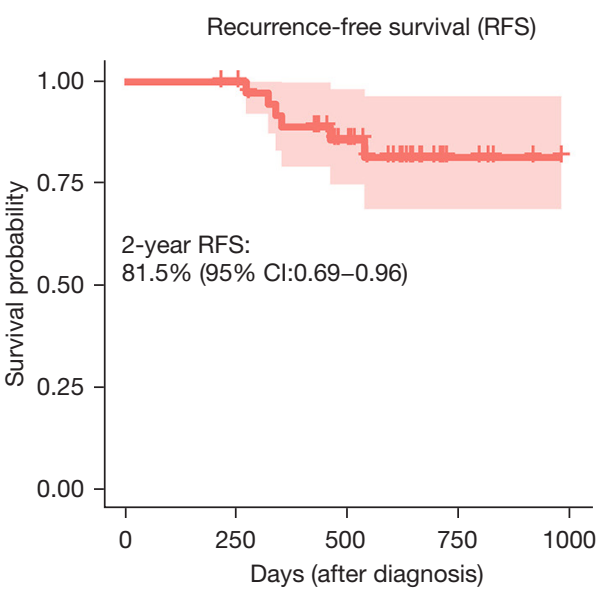

B

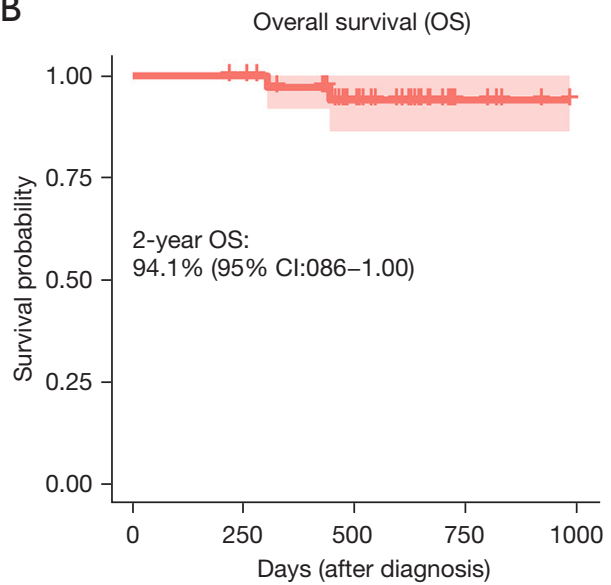

Figure 3 Survival analysis of patients receiving neoadjuvant immunotherapy in this cohort. (A,B) RFS and OS of patients receiving neoadjuvant immunotherapy in this cohort. RFS, recurrence-free survival; OS, overall survival.

predictive of the responsiveness to ICIs (24). Locally advanced NSCLC is typically detected as a large tumor that normally presents a high antigen burden with considerable clonal diversity, providing a rationale for immunotherapy in the neoadjuvant setting, in which a large tumor mass often does not allow for a complete resection by surgery alone.

In a preclinical study, Cascone and colleagues reported that in syngeneic NSCLC (344SQ) mouse models, mice were randomized to receive 3 doses of neoadjuvant immunoglobin g (IgG), anti-PD-1, anti-CTLA-4, antiPD-1 plus anti-CTLA-4, or observation alone (32). After treatment, primary tumors were resected. Subsequently, the observation group received 3 doses of adjuvant antiPD-1, anti-CTLA-4, or anti-PD-1 plus anti-CTLA-4. Final results showed that single ICI and combined ICI administration significantly improved survival as compared with the observation group in both neoadjuvant and adjuvant settings. More interestingly, further analysis showed that the combined therapy in the neoadjuvant setting produced longer survival than did combined therapy in the adjuvant setting (32), highlighting the importance of the neoadjuvant strategies in treating lung cancer.

Apart from the preclinical data, a recent single-arm clinical study conducted by Forde et al. (18) enrolled 20 patients with stage I-IIIA NSCLC who received 2 doses of neoadjuvant nivolumab every 2 weeks. The data showed that neoadjuvant nivolumab caused few side effects, did not delay surgery, and induced MPR in $45 \%$ of resected tumors. Interestingly, TMB was also found to be predictive of the pathological response to the PD-L1 antibody, nivolumab. More recently, based on preclinical evidence (32), Cascone et al. (33) conducted the first phase 2 randomized NEOSTAR trial to report on neoadjuvant nivolumab or nivolumab plus ipilimumab followed by surgery in 44 patients with stage I-III operable NSCLC. They found that combination therapy produced a significant clinical benefit, as assessed by the MPR rate (38\% vs. $22 \%)$. The evidence highlighted the superiority of neoadjuvant combined immunotherapy over single immunotherapy, which gives hopes for further investigation into operable NSCLC. However, in our study, we did not observe this tendency, which might be explained by the small sample size.

\section{Biomarkers predicting responses to immunotherapy}

Despite the promising results, biomarkers that predict the pathological responses to immunotherapy alone or chemo-immunotherapy are still lacking (9,34-36). This is a critical issue because the subsequent invasive surgery can be obviated in patients who have a pathological CR after ICI therapy. Neoadjuvant immunotherapy is an ideal setting for exploring predictive biomarkers, given that the pathologic responses can be directly and accurately assessed. By contrast, radiographic evaluation after ICI therapy is not reliable, while the radiographic mass can be still observed in some cases with $\mathrm{pMR}$ or $\mathrm{pCR}$.

Currently, PD-L1, TMB, and MSI are the only 3 clinically approved biomarkers that can predict benefit from ICI administration $(24,25)$. However, in this study, we found that PD-L1 expression evaluated by pretreatment biopsies was not associated with pathological response to ICIs (Table 2), 
which is in line with the results from clinical trial-based cohorts $(2,18,37)$. Interestingly, we found that smoking is an actionable biomarker to predict the pathological response to ICIs. Similar results have been reported $(2,38,39)$. In a retrospective analysis of NSCLC patients treated with any PD-1 inhibitor, the overall response rate was higher in heavy smokers (20.6\%) than in light or never smokers (4.2\%) (40). However, in another cohort involving 315 NSCLC patients with PD-L1 TPS $\geq 50 \%$ in 5 American academic medical centers, there were no significant differences in the objective responses among the never, light, and heavy smokers [never (27\%) vs. heavy (40\%), $\mathrm{P}=0.180$; light $(40 \%)$ vs. heavy; $\mathrm{P}=1.000]$ (41). Moreover, in one study, survival benefit favored ICIs in previously treated patients, but favored nonsmokers in treatment-naïve patients (42). More recently, increased smoking exposure had a significant association with improved clinical outcomes in metastatic NSCLC treated with ICI monotherapy independent of PD-L1 TPS (43). The inconsistency might be due to the response rates being evaluated via radiographic-based RECIST v1.1, which was shown to have a poor capacity to reflect the real pathological responses in our study (Figure 2).

PD-L1 represents the most commonly used clinical biomarkers to predict patients` response to immunotherapy. Previous evidence showed that smoking history was associated with increased PD-L1 expression in lung tumors (38). However, in this study, we did not observe this pattern. Instead, we found out that the smoking was likely to increase TMB/MSI, another two approved biomarkers for immunotherapy response, consequently increasing the response to immunotherapy (Figure $1 F, 1 G$ ). Supporting this notion, recent evidence also revealed the association between heavy smoking and TMB (26-28) and MSI $(29,30)$ in the corresponding tumors (Figure 1), since clinical trialbased evidence has demonstrated that TMB is linked to the pathological response to ICIs (18). Despite this, TMB is complex, and its related data are not available in real-time for decision-making in the first-line setting. By contrast, a heavy smoking history is much easier and more practical to use for stratifying NSCLC patients. These results highlight the possibility that heavy smokers may be prioritized for ICI or chemo-immunotherapy, which warrants further investigation.

There are some other factors within the tumor immune microenvironment that are closely associated with response to immunotherapy, suggesting involvement of complex mechanisms. Among those, TLSs, ectopic lymphoid organs that develop in non-lymphoid tissues at sites of chronic inflammation including tumours represent highly interesting components that could robustly predict the response to ICIs (44-46). In our study, we also examined the histopathology and observed some TLSs-like compartments in the samples with pCR (Figure 2).

\section{The performance of CT in the assessment of pathological response}

CT is routinely applied to assess the response to neoadjuvant treatment in patients with advanced NSCLC. Previous evidence has revealed that CT response in accordance with RECIST criteria could accurately predict OS in NSCLC patients after neoadjuvant chemotherapy (5). However, the discordance rate between histopathologic and CT scan-based response evaluation was more than $40 \%$ $(5,18)$. Moreover, it is difficult to judge the histopathologic response with a complete response by CT criteria. In the setting of neoadjuvant immunotherapy, we observed a high discrepancy between CT-based assessing therapy response after neoadjuvant immunotherapy versus histopathologic response after surgery, which might have been due to the mixture of infiltrative lymphocytes; stromal, fibrotic components; and tumor cell death within the tumor bed (Figure 2); leading to the inability of CT to accurately predict histopathologic response after neoadjuvant therapy $(47,48)$. In contrast, a heavy smoking history might be a promising predictor for high-degree (pCR/pMR) response to immunotherapy in the neoadjuvant setting (Tables 2,3; Figure 1) and should be studied further with a larger cohort.

\section{The role of surgery in the setting of neoadjuvant immunotherapy}

Neoadjuvant immunotherapy has just been investigated in patients with locally-advanced lung cancer. Despite the early promising response, whether the following surgery could provide additional benefits remains unknow. A welldesigned trial with long-term follow-up is required.

\section{The combined treatment with immunotherapy}

Despite the promises of immunotherapy alone for treating patients, highly heterogeneous response exists. Also, therapy resistance is common after ICIs treatment (49). Thus, combined systematic chemotherapy or other targeted therapies to synergize with immunotherapy has emerged recently (50). In this study cohort, some patients received chemo-immunotherapy; however, whether 
additional chemotherapy could provide synergistic efficacy remains to be defined due to the small sample size and short-term follow-up. Combined targeted therapies, such as CD73-adenosine (51,52), HDAC (epigenetic modulation) (53), tumor microenvironment (cancerassociated fibroblasts) (54), and metabolism (55) have been widely investigated, which, however, their clinical applications still have a long way to go.

\section{Limitations}

This study has some limitations, including inherent bias of a retrospective study with small sample size. Also, the patients were highly selected. Furthermore, we did not have the whole-genome sequencing data regarding other mutations (e.g., KEAP1, STK11) $(56,57)$ that relate to the response to ICIs. Validation with independent, multicenter cohorts and prospective studies is warranted.

\section{Conclusions}

In this lung cancer patient cohort receiving neoadjuvant immunotherapy plus surgery, the clinical outcomes favor lung cancer patients with a heavy smoking history, suggesting a smoking signature may be able to identify patient subsets that are highly responsive to neoadjuvant ICIs.

\section{Acknowledgments}

The authors appreciate the academic support from AME Lung Cancer Collaborative Group.

Funding: This research was funded by the National Natural Science Foundation of China (grant No. 82072570 to Feng Yao) and Medical-Engineering Interdisciplinary Research project of Shanghai Jiao Tong University (YG2021QN128 to Zhexin Wang).

\section{Footnote}

Reporting Checklist: The authors have completed the STARD reporting checklist. Available at https://dx.doi. org/10.21037/tlcr-21-734

Data Sharing Statement: Available at https://dx.doi. org/10.21037/tlcr-21-734

Conflicts of Interest: All authors have completed the ICMJE uniform disclosure form (available at https://dx.doi. org/10.21037/tlcr-21-734). FY reported that this study was funded by the National Natural Science Foundation of China (grant No. 82072570 to FY). ZW reported that this study was funded by Medical-Engineering Interdisciplinary Research project of Shanghai Jiao Tong University (YG2021QN128 to ZW). The other authors have no conflicts of interest to declare.

Etbical Statement: The authors are accountable for all aspects of the work in ensuring that questions related to the accuracy or integrity of any part of the work are appropriately investigated and resolved. All procedures performed in this study involving human participants were in accordance with the Declaration of Helsinki (as revised in 2013). The study was approved by the Institutional Review Board of Shanghai Chest Hospital [\#KS21039]. Informed consent was obtained from all participants involved in the study.

Open Access Statement: This is an Open Access article distributed in accordance with the Creative Commons Attribution-NonCommercial-NoDerivs 4.0 International License (CC BY-NC-ND 4.0), which permits the noncommercial replication and distribution of the article with the strict proviso that no changes or edits are made and the original work is properly cited (including links to both the formal publication through the relevant DOI and the license). See: https://creativecommons.org/licenses/by-nc-nd/4.0/.

\section{References}

1. Paz-Ares L, Luft A, Vicente D, et al. Pembrolizumab plus Chemotherapy for Squamous Non-Small-Cell Lung Cancer. N Engl J Med 2018;379:2040-51.

2. Gandhi L, Rodríguez-Abreu D, Gadgeel S, et al. Pembrolizumab plus Chemotherapy in Metastatic NonSmall-Cell Lung Cancer. N Engl J Med 2018;378:2078-92.

3. Duffy MJ, Crown J. Biomarkers for Predicting Response to Immunotherapy with Immune Checkpoint Inhibitors in Cancer Patients. Clin Chem 2019;65:1228-38.

4. Shembrey C, Huntington ND, Hollande F. Impact of Tumor and Immunological Heterogeneity on the AntiCancer Immune Response. Cancers (Basel) 2019;11:1217.

5. William WN Jr, Pataer A, Kalhor N, et al. Computed tomography RECIST assessment of histopathologic response and prediction of survival in patients with resectable non-small-cell lung cancer after neoadjuvant chemotherapy. J Thorac Oncol 2013;8:222-8. 
6. Cottrell TR, Thompson ED, Forde PM, et al. Pathologic features of response to neoadjuvant anti-PD-1 in resected non-small-cell lung carcinoma: a proposal for quantitative immune-related pathologic response criteria (irPRC). Ann Oncol 2018;29:1853-60.

7. Travis WD, Dacic S, Wistuba I, et al. IASLC Multidisciplinary Recommendations for Pathologic Assessment of Lung Cancer Resection Specimens After Neoadjuvant Therapy. J Thorac Oncol 2020;15:709-40.

8. Reuss JE, Anagnostou V, Cottrell TR, et al. Neoadjuvant nivolumab plus ipilimumab in resectable non-small cell lung cancer. J Immunother Cancer 2020;8:e001282.

9. Jiang L, Huang J, Jiang S, et al. The surgical perspective in neoadjuvant immunotherapy for resectable nonsmall cell lung cancer. Cancer Immunol Immunother 2021;70:2313-21.

10. Yang ZR, Liu MN, Yu JH, et al. Treatment of stage III non-small cell lung cancer in the era of immunotherapy: pathological complete response to neoadjuvant pembrolizumab and chemotherapy. Transl Lung Cancer Res 2020;9:2059-73.

11. Ren S, Wang C, Shen J, et al. Neoadjuvant immunotherapy with resectable non-small cell lung cancer: recent advances and future challenges. J Thorac Dis 2020;12:1615-20.

12. Dickhoff C, Senan S, Schneiders FL, et al. Ipilimumab plus nivolumab and chemoradiotherapy followed by surgery in patients with resectable and borderline resectable T34N0-1 non-small cell lung cancer: the INCREASE trial. BMC Cancer 2020;20:764.

13. Rossi G, Russo A, Tagliamento M, et al. Precision Medicine for NSCLC in the Era of Immunotherapy: New Biomarkers to Select the Most Suitable Treatment or the Most Suitable Patient. Cancers (Basel) 2020;12:1125.

14. Jin J, Yang L, Liu D, et al. Association of the neutrophil to lymphocyte ratio and clinical outcomes in patients with lung cancer receiving immunotherapy: a meta-analysis. BMJ Open 2020;10:e035031.

15. Detterbeck FC, Boffa DJ, Kim AW, et al. The Eighth Edition Lung Cancer Stage Classification. Chest 2017;151:193-203.

16. Fernandez FG, Falcoz PE, Kozower BD, et al. The Society of Thoracic Surgeons and the European Society of Thoracic Surgeons general thoracic surgery databases: joint standardization of variable definitions and terminology. Ann Thorac Surg 2015;99:368-76.

17. Schwartz LH, Litière $S$, de Vries E, et al. RECIST 1.1-Update and clarification: From the RECIST committee. Eur J Cancer 2016;62:132-7.

18. Forde PM, Chaft JE, Smith KN, et al. Neoadjuvant PD-1 Blockade in Resectable Lung Cancer. N Engl J Med 2018;378:1976-86.

19. Lee YH, Shin MH, Kweon SS, et al. Cumulative smoking exposure, duration of smoking cessation, and peripheral arterial disease in middle-aged and older Korean men. BMC Public Health 2011;11:94.

20. Yang H, Liang SQ, Xu D, et al. HSP90/AXL/eIF4Eregulated unfolded protein response as an acquired vulnerability in drug-resistant KRAS-mutant lung cancer. Oncogenesis 2019;8:45.

21. Kautto EA, Bonneville R, Miya J, et al. Performance evaluation for rapid detection of pan-cancer microsatellite instability with MANTIS. Oncotarget 2017;8:7452-63.

22. Samstein RM, Lee CH, Shoushtari AN, et al. Tumor mutational load predicts survival after immunotherapy across multiple cancer types. Nat Genet 2019;51:202-6.

23. Yang H, Xu J, Yao F, et al. Analysis of unexpected small cell lung cancer following surgery as the primary treatment. J Cancer Res Clin Oncol 2018;144:2441-7.

24. Wan L, Wang Z, Xue J, et al. Tumor mutation burden predicts response and survival to immune checkpoint inhibitors: a meta-analysis. Transl Cancer Res 2020;9:5437-49.

25. André T, Shiu KK, Kim TW, et al. Pembrolizumab in Microsatellite-Instability-High Advanced Colorectal Cancer. N Engl J Med 2020;383:2207-18.

26. Wang X, Ricciuti B, Nguyen T, et al. Association between Smoking History and Tumor Mutation Burden in Advanced Non-Small Cell Lung Cancer. Cancer Res 2021;81:2566-73.

27. Stabile LP, Kumar V, Gaither-Davis A, et al. Syngeneic tobacco carcinogen-induced mouse lung adenocarcinoma model exhibits PD-L1 expression and high tumor mutational burden. JCI Insight 2021;6:145307.

28. Nagahashi M, Sato S, Yuza K, et al. Common driver mutations and smoking history affect tumor mutation burden in lung adenocarcinoma. J Surg Res 2018;230:181-5.

29. Doukas SG, Vageli DP, Lazopoulos G, et al. The Effect of NNK, A Tobacco Smoke Carcinogen, on the miRNA and Mismatch DNA Repair Expression Profiles in Lung and Head and Neck Squamous Cancer Cells. Cells 2020;9:1031.

30. Warth A, Körner S, Penzel R, et al. Microsatellite instability in pulmonary adenocarcinomas: a 
comprehensive study of 480 cases. Virchows Arch 2016;468:313-9.

31. Jiang T, Shi T, Zhang H, et al. Tumor neoantigens: from basic research to clinical applications. J Hematol Oncol 2019;12:93.

32. Cascone T, Hamdi H, Zhang F, et al. Abstract 1719: Superior efficacy of neoadjuvant compared to adjuvant immune checkpoint blockade in non-small cell lung cancer. Cancer Res 2018;78:1719.

33. Cascone T, William WN Jr, Weissferdt A, et al. Neoadjuvant nivolumab or nivolumab plus ipilimumab in operable non-small cell lung cancer: the phase 2 randomized NEOSTAR trial. Nat Med 2021;27:504-14.

34. Pradhan M, Chocry M, Gibbons DL, et al. Emerging biomarkers for neoadjuvant immune checkpoint inhibitors in operable non-small cell lung cancer. Transl Lung Cancer Res 2021;10:590-606.

35. Ling Y, Li N, Li L, et al. Different pathologic responses to neoadjuvant anti-PD-1 in primary squamous lung cancer and regional lymph nodes. NPJ Precis Oncol 2020;4:32.

36. Gao P, Chen $\mathrm{H}$, Zeng T, et al. A pathological complete response to neoadjuvant chemotherapy and immunotherapy in a non-small cell lung cancer patient. Transl Lung Cancer Res 2020;9:2157-60.

37. Gadgeel S, Rodríguez-Abreu D, Speranza G, et al. Updated Analysis From KEYNOTE-189: Pembrolizumab or Placebo Plus Pemetrexed and Platinum for Previously Untreated Metastatic Nonsquamous Non-Small-Cell Lung Cancer. J Clin Oncol 2020;38:1505-17.

38. Li JJN, Karim K, Sung M, et al. Tobacco exposure and immunotherapy response in $\mathrm{PD}-\mathrm{L} 1$ positive lung cancer patients. Lung Cancer 2020;150:159-63.

39. Ng TL, Liu Y, Dimou A, et al. Predictive value of oncogenic driver subtype, programmed death-1 ligand (PD-L1) score, and smoking status on the efficacy of PD-1/PD-L1 inhibitors in patients with oncogene-driven non-small cell lung cancer. Cancer 2019;125:1038-49.

40. Gainor JF, Shaw AT, Sequist LV, et al. EGFR Mutations and ALK Rearrangements Are Associated with Low Response Rates to PD-1 Pathway Blockade in Non-Small Cell Lung Cancer: A Retrospective Analysis. Clin Cancer Res 2016;22:4585-93.

41. Gainor JF, Rizvi H, Jimenez Aguilar E, et al. Clinical activity of programmed cell death 1 (PD-1) blockade in never, light, and heavy smokers with non-small-cell lung cancer and PD-L1 expression $\geq 50$. Ann Oncol 2020;31:404-11.
42. Norum J, Nieder C. Tobacco smoking and cessation and PD-L1 inhibitors in non-small cell lung cancer (NSCLC): a review of the literature. ESMO Open 2018;3:e000406.

43. Wang X, Ricciuti B, Alessi JV, et al. Smoking History as a Potential Predictor of Immune Checkpoint Inhibitor Efficacy in Metastatic Non-Small Cell Lung Cancer. J Natl Cancer Inst 2021. [Epub ahead of print]. doi: 10.1093/jnci/ djab116.

44. Cabrita R, Lauss M, Sanna A, et al. Tertiary lymphoid structures improve immunotherapy and survival in melanoma. Nature 2020;577:561-5.

45. Helmink BA, Reddy SM, Gao J, et al. B cells and tertiary lymphoid structures promote immunotherapy response. Nature 2020;577:549-55.

46. Petitprez F, de Reyniès A, Keung EZ, et al. B cells are associated with survival and immunotherapy response in sarcoma. Nature 2020;577:556-60.

47. Lee HY, Lee HJ, Kim YT, et al. Value of combined interpretation of computed tomography response and positron emission tomography response for prediction of prognosis after neoadjuvant chemotherapy in non-small cell lung cancer. J Thorac Oncol 2010;5:497-503.

48. Yang H, Sun B, Xu K, et al. Pharmaco-transcriptomic correlation analysis reveals novel responsive signatures to HDAC inhibitors and identifies Dasatinib as a synergistic interactor in small-cell lung cancer. EBioMedicine 2021;69:103457.

49. Jackson CM, Choi J, Lim M. Mechanisms of immunotherapy resistance: lessons from glioblastoma. Nat Immunol 2019;20:1100-9.

50. Li S, Chu X, Ye L, et al. A narrative review of synergistic drug administration in unresectable locally advanced non-small cell lung cancer: current landscape and future prospects in the era of immunotherapy. Transl Lung Cancer Res 2020;9:2082-96.

51. Allard D, Allard B, Gaudreau PO, et al. CD73adenosine: a next-generation target in immuno-oncology. Immunotherapy 2016;8:145-63.

52. Yang H, Yao F, Davis PF, et al. CD73, Tumor Plasticity and Immune Evasion in Solid Cancers. Cancers (Basel) 2021;13:177.

53. Park J, Thomas S, Munster PN. Epigenetic modulation with histone deacetylase inhibitors in combination with immunotherapy. Epigenomics 2015;7:641-52.

54. Barrett RL, Puré E. Cancer-associated fibroblasts and their influence on tumor immunity and immunotherapy. Elife 2020;9:57243. 
55. DePeaux K, Delgoffe GM. Metabolic barriers to cancer immunotherapy. Nat Rev Immunol 2021. [Epub ahead of print]. doi: 10.1038/s41577-021-00541-y.

56. Skoulidis F, Goldberg ME, Greenawalt DM, et al. STK11/LKB1 Mutations and PD-1 Inhibitor Resistance

Cite this article as: Yang H, Ma W, Sun B, Fan L, Xu K, Hall SRR, Al-Hurani MF, Schmid RA, Peng RW, Hida T, Wang Z, Yao F. Smoking signature is superior to programmed deathligand 1 expression in predicting pathological response to neoadjuvant immunotherapy in lung cancer patients. Transl Lung Cancer Res 2021;10(9):3807-3822. doi: 10.21037/tlcr21-734 in KRAS-Mutant Lung Adenocarcinoma. Cancer Discov 2018;8:822-35.

57. Yang H, Liang SQ, Schmid RA, et al. New Horizons in KRAS-Mutant Lung Cancer: Dawn After Darkness. Front Oncol 2019;9:953. 
Supplementary

Table S1 Immunotherapy or chemo-immunotherapy regimens used in this study

Immunotherapy regimens

No.

$\mathrm{ICI}(\mathrm{s})$ alone

Pembrolizumab

Nivolumab

3

Nivolumab + ipilimumab

Sintilimab

Single $\mathrm{ICl}$ plus platinum doublets

Pembrolizumab + paclitaxel plus platinum

Pembrolizumab + pemetrexed plus platinum

Pembrolizumab + gemcitabine plus platinum

Nivolumab + docetaxel plus platinum

1

Nivolumab + paclitaxel plus platinum

Nivolumab + pemetrexed plus platinum

2

Nivolumab + gemcitabine plus platinum

4

Sintilimab + paclitaxel + carboplatin

Sintilimab + docetaxel plus platinum

$\mathrm{ICl}$, immune checkpoint inhibitors. 


\section{Patient \#1, LUSC}
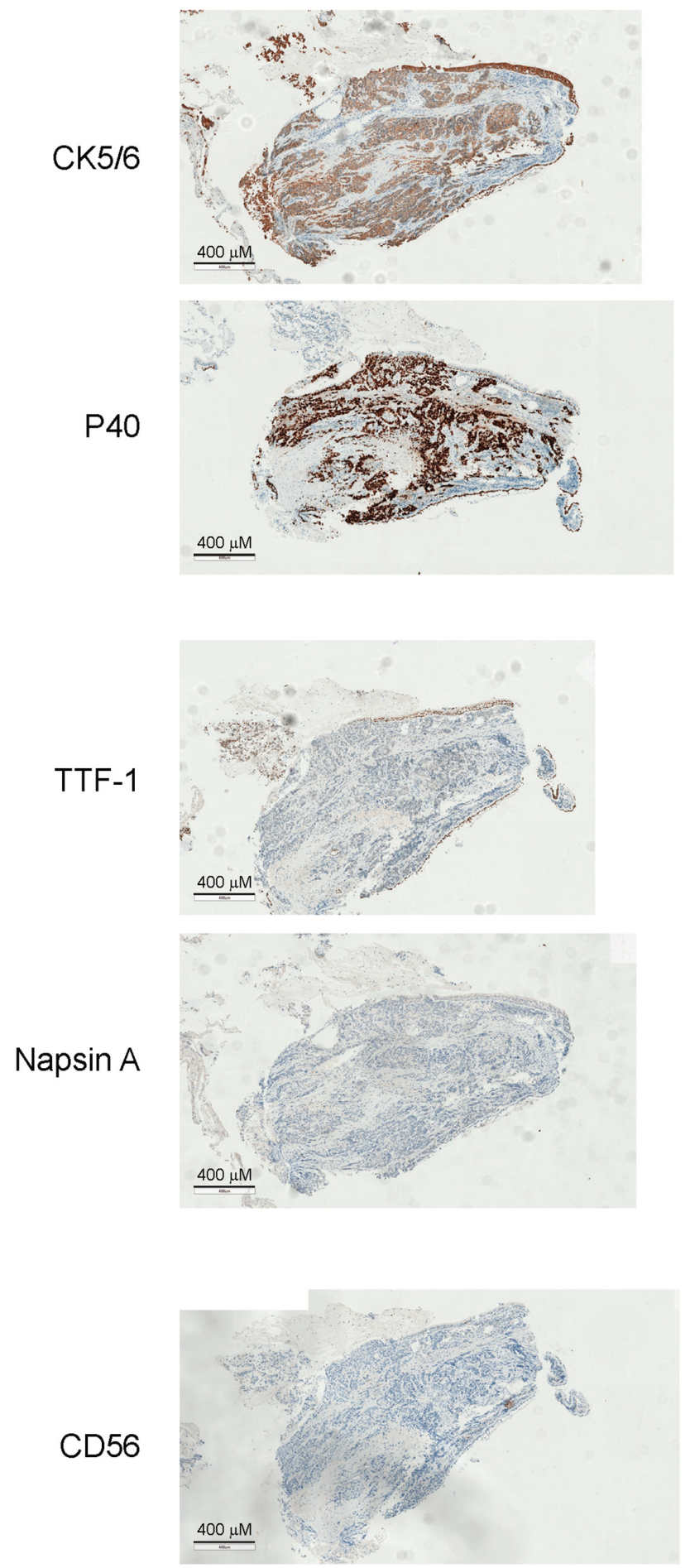

\section{Patient \#2, LUSC}
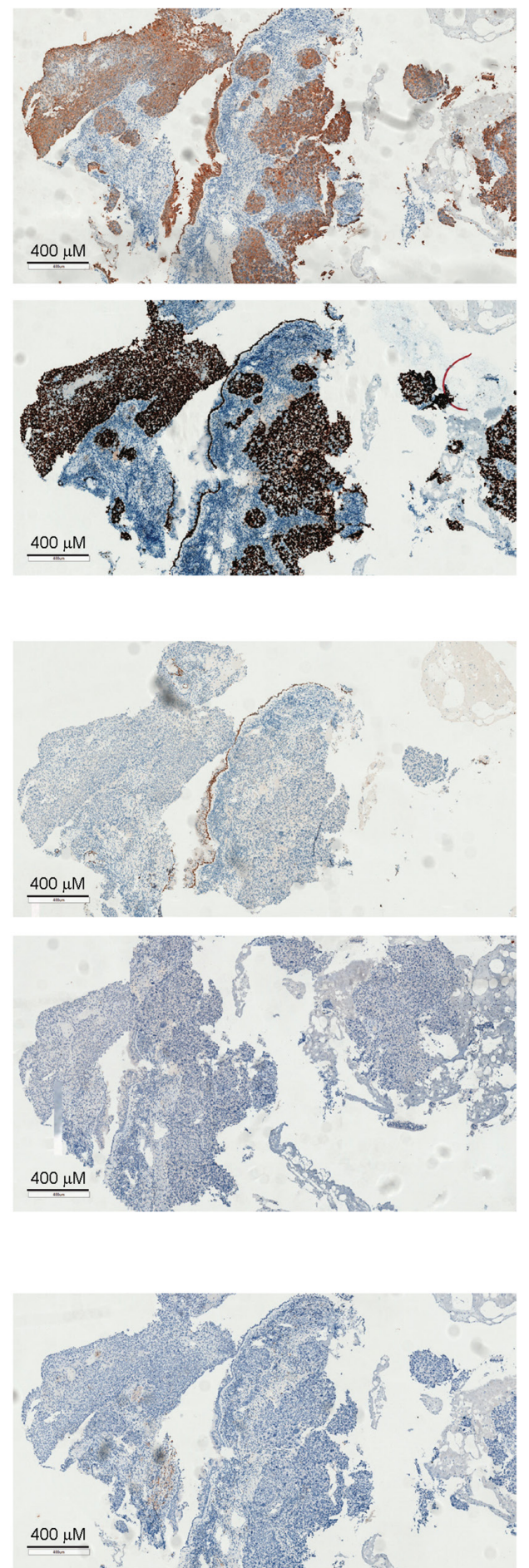

Figure S1 Immunohistochemistry staining of biopsy samples before neoadjuvant therapy. Scale bar, $400 \mu \mathrm{M}$. LUSC, lung squamous cell carcinoma. 